\title{
Efficient Discovery of Spectrum Opportunities with MAC-Layer Sensing in Cognitive Radio Networks
}

\author{
Hyoil Kim and Kang G. Shin, Fellow, IEEE
}

\begin{abstract}
Sensing/monitoring of spectrum-availability has been identified as a key requirement for dynamic spectrum allocation in cognitive radio networks (CRNs). An important issue associated with MAC-layer sensing in CRNs is how often to sense the availability of licensed channels and in which order to sense those channels. To resolve this issue, we address 1) how to maximize the discovery of spectrum opportunities by sensing-period adaptation and 2) how to minimize the delay in finding an available channel. Specifically, we develop a sensing-period optimization mechanism and an optimal channel-sequencing algorithm, as well as an environmentadaptive channel-usage pattern estimation method. Our simulation results demonstrate the efficacy of the proposed schemes and its significant performance improvement over nonoptimal schemes. The sensing-period optimization discovers more than 98 percent of the analytical maximum of discoverable spectrum-opportunities, regardless of the number of channels sensed. For the scenarios tested, the proposed scheme is shown to discover up to 22 percent more opportunities than nonoptimal schemes, which may become even greater with a proper choice of initial sensing periods. The idle-channel discovery delay with the optimal channel-sequencing technique ranges from 0.08 to 0.35 seconds under the tested scenarios, which is much faster than nonoptimal schemes. Moreover, our estimation method is shown to track time-varying channel-parameters accurately.
\end{abstract}

Index Terms-Cognitive radios, spectrum agility, spectrum opportunity, spectrum sensing, channel-usage patterns.

\section{INTRODUCTION}

$\mathrm{T}$ HERE have been numerous protocol standards on the wireless spectrum that rely on a static spectrum allocation policy under which each licensed spectrum band is statically assigned to the specific licensed service and its users. Once a spectrum band is assigned to a certain service, its allocation is not allowed to change. However, a new concept of dynamic spectrum allocation has become necessary to overcome critical limitations of the traditional staticallocation scheme. Recent studies have shown that the use of static spectrum allocation has degraded spectral efficiency significantly [1]. Moreover, current standards cannot guarantee the prevention of unexpected interruptions by wireless network users [2].

To alleviate these problems, FCC has recently suggested a new concept of cognitive radio networks (CRNs) that serves as a framework in realizing dynamic spectrum allocation. It requires the enhancement of current PHY and MAC protocols to adopt spectrum-agile features. The basic idea of spectrum agility is to allow secondary users (SUs) or unlicensed users to access licensed spectrum bands ${ }^{1}$ as far as they do not incur any harmful interference with the

1. The terms spectrum band and channel are used interchangeably throughout this paper.

- The authors are with the Department of Electrical Engineering and Computer Science, The University of Michigan, Computer Science and Engineering Building, 2260 Hayward Street, Ann Arbor, MI 48109-2121. E-mail: \{hyoilkim,kgshin\}@eecs.umich.edu.

Manuscript received 31 Dec. 2006; revised 23 May 2007; accepted 30 July 2007; published online 22 Aug. 2007.

For information on obtaining reprints of this article, please send e-mail to: tmc@computer.org, and reference IEEECS Log Number TMC-0350-1206.

Digital Object Identifier no. 10.1109/TMC.2007.70751. primary users (PUs) or licensed users of the bands. To achieve this goal, SUs must monitor each channel's usage pattern by its PUs to identify spectrum holes or opportunities [3] to exploit. Whenever SUs find a channel that can be utilized without interfering with its PUs, it can be assigned to and will be shared by the SUs. The SUs are also responsible for monitoring returning PUs on the channel they are currently using so as to promptly vacate the channel in such a case.

This new framework of spectrum access has been given different names, such as the Dynamic Spectrum Access (DSA) [4] or neXt Generation (XG) [5], [6] protocol. Sensing the status/availability of a channel is commonly recognized as one of the most fundamental elements of a CR (and, hence, this framework) due to its crucial role of discovering spectrum opportunities and detecting the existence/return of PUs [5], [6], [7]. Spectrum sensing can be realized as a two-layer mechanism. The PHY-layer sensing focuses on efficiently detecting PU signals to identify opportunities by adapting its modulation/encoding schemes and parameters. Several well-known PHY-layer detection methods such as energy detection, matched filter, and feature detection [8], [9], [10] have been proposed as candidates for the PHYlayer sensing. On the other hand, the MAC-layer sensing determines when SUs have to sense which channels. This type of sensing, despite its importance, has received far less attention than other related topics. Recently, many MAClayer researchers have considered fair channel allocation and SUs coordination under the assumption of the availability of sensing results. However, they have not considered how the MAC-layer sensing works and how to 
schedule sensing for efficient discovery of spectrum opportunities.

In this paper, we focus on two important issues of the MAC-layer sensing: 1) how to maximize the overall discovery of opportunities in the licensed channels and 2) how to minimize the delay in locating an idle channel in case a secondary network (SN; a group of SUs) has to actively search for an idle channel. We propose two novel schemes. The first is the sensing-period optimization that maximizes the discovery of opportunities from licensed channels. The second is the optimal channel-sequencing algorithm that minimizes delay in searching for an idle channel. To support the two proposed schemes, we also introduce an estimation method for characterizing a channel's usage pattern by its PUs.

The rest of the paper is organized as follows: Section 2 presents the network/channel models and our assumptions. Section 3 describes the motivations of our work. Section 4 presents the sensing-period optimization technique to achieve maximum discovery of opportunities and Section 5 describes how to determine the optimal sensing sequence of channels so as to minimize the delay in locating an idle channel. Section 6 introduces the channel-usage pattern estimation method. The Matlab-based simulation results are presented and analyzed in Section 7. Example deployment scenarios of the proposed schemes are discussed in Section 8. Section 9 discusses other related work. Finally, we conclude the paper and suggest future directions in Section 10.

\section{Preliminaries}

\subsection{Network Topology}

A group of SUs is assumed to form a single-hop wireless SN within the transmission range of which there are no other SNs interfering or cooperating with that $\mathrm{SN}$. In a practical CRN, however, such as an IEEE 802.22 network [11], the interference among adjacent SNs should be dealt with in the context of internetwork coordination of channel sensing and allocation. Although the coordination issue is not the main focus of this paper, our proposed scheme can coexist with any coordination scheme by dynamically adapting the pool of available channels for an SN in such a way that those channels are not used simultaneously by other SNs.

Every $\mathrm{SU}$ in the SN is assumed to be equipped with a single identical antenna that can be tuned to any combination of $N$ consecutive licensed channels. This can be done by the Orthogonal Frequency Division Multiplexing (OFDM) technique with adaptive and selective allocation of OFDM subcarriers to utilize any subset of $N$ licensed channels at the same time [12], [13], [14]. Note that equipping each $\mathrm{SU}$ with more than one antenna might cause severe interference among its antennas, thus degrading the SU's performance [15]. We therefore focus on SUs, each equipped with a single antenna. Each SU works as a transceiver, as well as a sensor in its SN.

An important role of sensing is incumbent detection, that is, determining the presence/absence of PUs on a channel. Energy and feature detections are two prominent PHYsensing schemes for incumbent detection. Energy detection, however, cannot differentiate PU signals from SU signals since it only measures the energy of a signal. Although feature detection can be used to overcome this difficulty, it may be harder to detect PU signals if SU signals interfere with them during sensing. Hence, IEEE 802.22 introduced the concept of a quiet period [11], [16] during which all SUs should suspend their transmission so that any sensor monitoring the channel may observe the presence/absence of PU signals without interference. It is assumed that a channel will be sensed within a quiet period whose schedule should be negotiated/reserved among SUs.

It is also assumed that all SUs in an SN should participate in sensing a channel at the same time for each scheduled measurement period to enhance the detection of PU signals even in a fading/shadowing environment. Fading/shadowing is known to become a serious problem in achieving the desirable sensing quality in terms of incumbent detection and false alarm probabilities [17], [18], [19]. To overcome these difficulties, collaborative sensing has been proposed, which requires multiple sensors to cooperate. Note, however, that this is not our focus, and hence, we will use a basic collaboration policy of letting all SUs participate in simultaneously sensing a channel.

\subsection{Channel-Usage Model}

Spectrum sensing mainly aims to check a channel's availability. Depending on its PUs' usage pattern, a channel could be modeled as an ON-OFF source alternating between ON (busy) and OFF (idle) periods. Such a model captures the time period in which the channel can be utilized by SUs without causing any harmful interference to PUs. Once an OFF period is discovered, SUs can utilize any portion of the remaining OFF period for their own transmission.

For channel $i(i=1,2, \ldots, N){ }^{2}$ we model the sojourn time of an $\mathrm{ON}$ period as a random variable $T_{O N}^{i}$ with the probability density function (p.d.f.) $f_{T_{O N}^{i}}(y), y>0$. Similarly, the p.d.f. of the sojourn time in an OFF period is given as $f_{T_{O F F}^{i}}(x), x>0 .{ }^{3} \mathrm{ON}$ periods are assumed to be independent and identically distributed (i.i.d.) and so are OFF periods. We also assume that $\mathrm{ON}$ and OFF periods are independent of each other.

Let $Z^{i}(t)$ denote the state (ON/OFF) of channel $i$ at time $t$. Then, $\left\{Z^{i}(t), t \geq 0\right\}$ becomes a semi-Markov process in that whenever the process enters ON/OFF state, the time until the next state transition is governed by p.d.f. $f_{T_{O N}^{i}}(y) / f_{T_{O F F}^{i}}(x)$. Since there are only two possible states, the behavior of this process can be analyzed by using the theory of alternating renewal processes [20], [21]. Fig. 1 shows the state transition model of this semiMarkov process.

Sensing is nothing but a sampling procedure of the given channel process $\left\{Z^{i}(t), t \geq 0\right\}$ to discover its state at each sensing instant. Let a sample from an ON/OFF period correspond to the value $1 / 0$. Then, sensing produces a binary random sequence for each channel. Fig. 2 illustrates periodic sensing with sensing period $T_{P}^{i}$ and sensing time $T_{I}^{i}$. Here, $T_{I}^{i}$ is a predefined amount of time for a single measurement in order to achieve the desirable level

2. We will use $i$ as the channel index throughout the paper.

3. Note that ON/OFF periods are not necessarily exponentially distributed. 


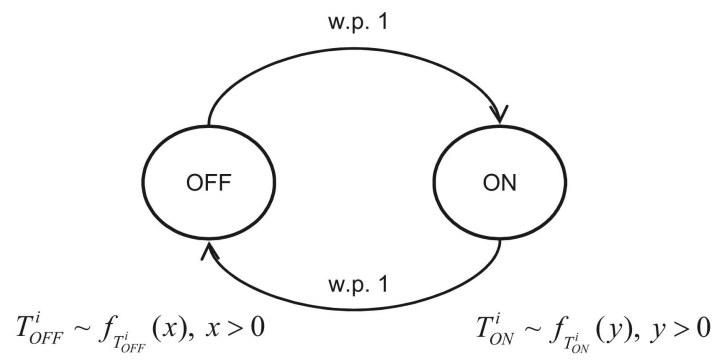

Fig. 1. The state-transition diagram of the semi-Markov process.

of detection quality by PHY-layer sensing. For example, it has been proposed in the IEEE 802.22 standard [16] that less than $1 \mathrm{~ms}$ should be spent for fast sensing with energy detection. We assume that $T_{I}^{i}$ is predetermined by PHYlayer sensing, and it is small relative to $E\left[T_{O F F}^{i}\right]$ and $E\left[T_{O N}^{i}\right]$.

On the other hand, channel utilization $u^{i}$, which is defined as the fraction of time in which channel $i$ is in ON state, is given as $u^{i}=E\left[T_{O N}^{i}\right] /\left(E\left[T_{O N}^{i}\right]+E\left[T_{O F F}^{i}\right]\right)$.

\subsection{Opportunity-Usage Model}

Opportunity represents a spectrum hole (that is, an OFF period) in a licensed channel. An opportunity in a channel can only be discovered by sensing the channel. As discussed earlier, it is necessary to perform collaborative sensing to overcome uncertainties in a wireless spectrum such as fading/shadowing. In collaborative sensing, the sample of a channel collected by an SU must be shared/ synchronized with other SUs so that each SU can decide on the channel's availability. Ghasemi and Sousa [17] introduced a simple rule (OR rule) in which a channel is considered $\mathrm{ON}$ if at least one $\mathrm{SU}$ reports that the channel is busy. Since the cooperation among SUs is not a focus of this paper, we assume that the sensing time $T_{I}^{i}$ includes both PHY-layer detection time (for example, $1 \mathrm{~ms}$ ) and data synchronization time in collaborative sensing.

Whenever sensing is performed on a channel and an opportunity on the channel is discovered, the channel is merged into a pool of available channels where the pool is called a logical channel. Therefore, a logical channel can include $0 \sim N$ licensed channels depending on their availability at that instant. The logical channel is treated as if it were a single channel whose capacity is equal to the sum of all licensed channels merged into it. This can be done by using the OFDM technique with selective allocation of subcarriers to the channels to be utilized [12], [13], [14]. In this way, more than one (possibly nonadjacent) channel in the logical channel can be simultaneously utilized by a single SU. The term home channel will be used to represent a licensed channel which is merged into the logical channel and being utilized by SUs. In contrast, a channel that does not belong to the logical channel is called a foreign channel.

For SUs to share the logical channel, we assume the following medium-access model: 1) SUs with packets to transmit compete with each other to gain exclusive access to the logical channel, 2) while an SU is transmitting, other SUs keep silent, and 3) the SU who has gained exclusive access to the channel should listen to the medium before transmission to detect returning PUs (Listen-Before-Talk policy [12]).

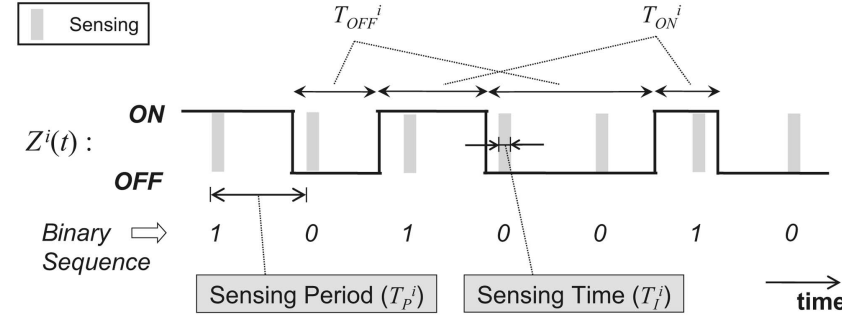

Fig. 2. The illustration of sensing of an ON-OFF alternating channel.

The return of PUs on a home channel should be detected promptly to minimize interference on them. This can be done by the Listen-before-Talk policy where every SU has to listen to the medium before commencing any packet transmission. Hence, we can assume that returning PUs can be detected within a reasonably small amount of time so that the channel can be vacated by SUs promptly. To vacate the channel due to returning PUs, OFDM should reconfigure subcarriers to exclude the channel band from usage. As a special case, if the home channel to be vacated is the only member of the logical channel, there will be no more channels to utilize. We call this situation channel switching in that the SUs should switch from the current channel (where its PUs just returned) to a new idle channel (which should be discovered). It is important to find the new idle channel as soon as possible so that SUs can resume their data transmission with the least interruption.

\section{Motivations}

\subsection{Maximal Discovery of Opportunities}

When the bandwidth requirement by an $\mathrm{SN}$ can be met with a single home channel, it would suffice to discover one idle channel and stay on the channel without searching for others until the channel is reclaimed by its PUs. Hence, sensing is performed on demand only when the SN must switch to another channel, and this type of sensing is called reactive sensing. An example of reactive sensing is illustrated in Fig. 3.

In this paper, we are interested in the case of SUs seeking more bandwidth than just a single home channel. With more home channels in a logical channel, an SU may transmit packets at a higher data rate. To discover more idle channels, foreign channels must be proactively sensed. That is, each channel should be sensed periodically with its own sensing period $T_{P}^{i}$. Any idle channel discovered by the periodic sampling becomes a new member of the logical channel, which can then provide more bandwidth. Although the periodic sensing is performed on every channel independently, the concurrent sensing of $N$ channels must be scheduled in such a way that there would be no other scheduled sensing while a measurement on channel $i$ is being performed for $T_{I}^{i}$ seconds. This type of sensing is called proactive sensing, an example of which is shown in Fig. 4 . The proactive sensing is formed by periodic sensing, as well as on-demand sensing. Note that on-demand sensing is the common part for both reactive and proactive sensing since channel switching occurs in either case. Another benefit of proactive sensing is that it finds an idle channel (at 

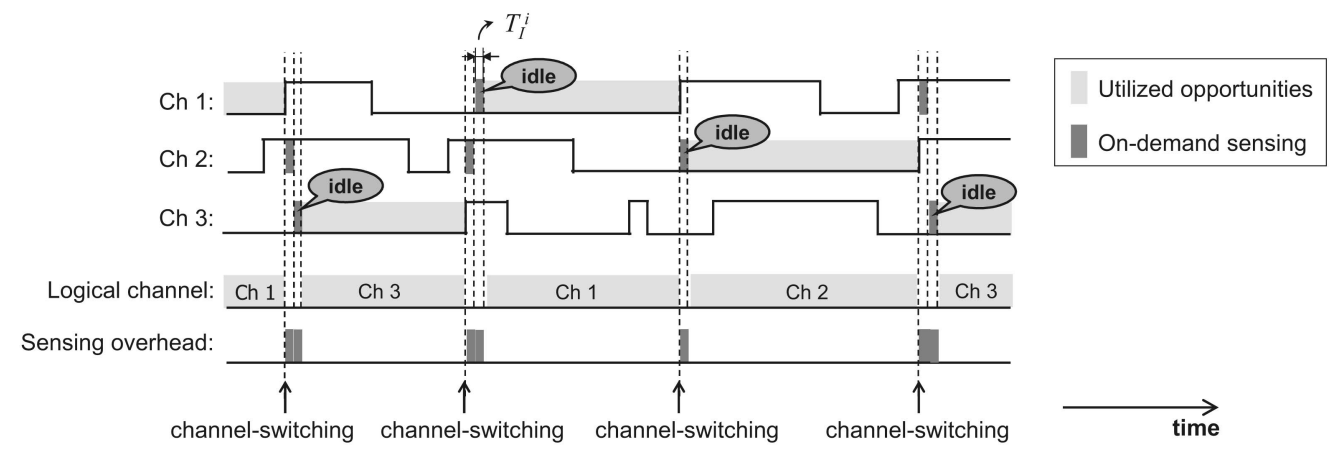

Fig. 3. An example of reactive sensing.

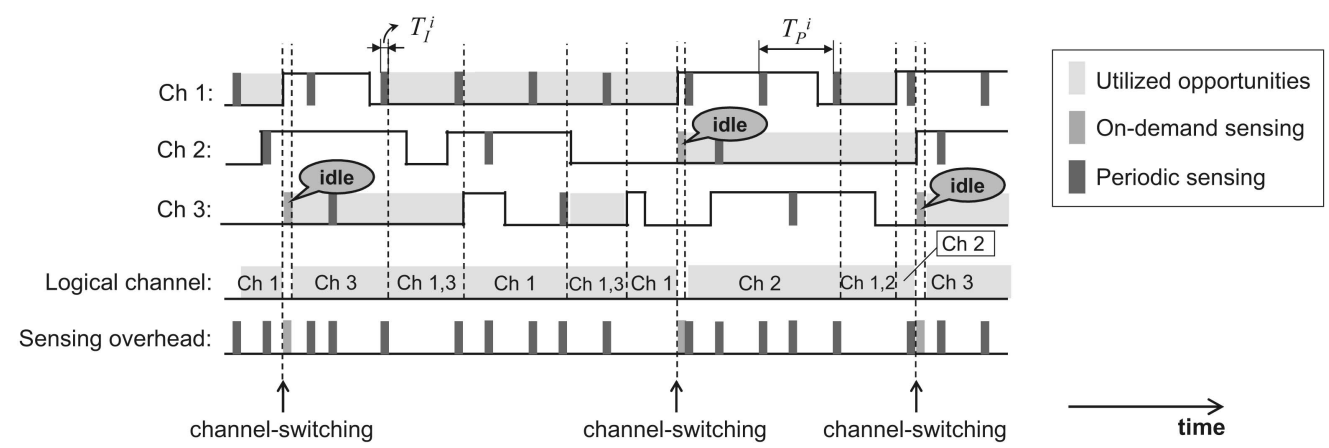

Fig. 4. An example of proactive sensing.

the time of channel switching) faster than reactive sensing because the former has more information (that is, periodic samples) on channels.

In general, more frequent sensing discovers more opportunities. However, one has to account for the fact that each sensing costs an SU $T_{I}^{i}$ time units without any packet transmission on the discovered opportunities since there is only a single antenna for both sensing and transmission. Hence, there is a trade-off between sensingtime overhead and discovery of opportunities. In Section 4, we propose a sensing-period optimization for the proactive sensing by making the trade-off between the discovered opportunities and the sensing overhead.

\subsection{Minimum Channel-Switching Latency}

When an SN needs to switch channels, it must discover a new opportunity with minimum delay so that SUs in the network can resume their communication quickly. Channel switching occurs in both reactive and proactive sensing, although the switching frequency may be different. Hence, it is important for CRNs to minimize the delay in finding an idle channel at the time of channel switching.

Upon triggering a channel switch (due to the return of PUs), the SN starts to search for a new home channel. Since we have assumed a simple collaborative sensing scheme, which makes all SUs to participate in sensing a channel simultaneously, $(N-1)$ foreign channels cannot be searched at the same time, and hence, they must be sensed sequentially. It is therefore important to know the optimal sensing sequence to minimize the searching delay. In Section 5, we propose the optimal sensing-sequencing algorithm to achieve the minimum channel-switching latency (CSL) by estimating the ON-OFF pattern of foreign channels. Here, the CSL is defined as the time elapsed since triggering of a channel switch until the discovery of the first idle channel.

\subsection{Estimation of Channel-Usage Pattern}

The motivations discussed so far depend on the underlying channel-usage patterns, which we model as ON-OFF alternating processes. Hence, the key is to estimate distribution parameters of ON/OFF periods to formulate our objective functions. Section 6 introduces an estimation procedure that can track time-varying parameters.

\section{Maximal Discovery of Opportunities by Optimizing Sensing Period}

When proactive sensing is employed by an $\mathrm{SN}$ and each channel is sensed periodically with its own sensing period, we would like to optimize the set of $N$ sensing periods $\left(T_{P}^{i} \mathrm{~S}\right)$ to maximize the discovery of opportunities.

Since sensing is nothing but a sampling process, it is not possible to exactly identify each state transition between $\mathrm{ON}$ and OFF periods. Hence, the time portion of a discovered OFF period between the start time and the discovery time of the OFF period cannot be utilized. In addition, some OFF periods may remain undiscovered at all if sensing is infrequent. However, blindly increasing the sensing frequency is not desirable, as it will increase the sensing overhead, which is proportional to the sum of $\left(T_{I}^{i} / T_{P}^{i}\right)$. Note that the sensing overhead is the time overhead during which all data traffic among SUs must be suspended to measure a channel's availability. This trade-off must be captured in the construction of an equation to find the optimal sensing frequencies/periods. Therefore, for each channel $i$, we 


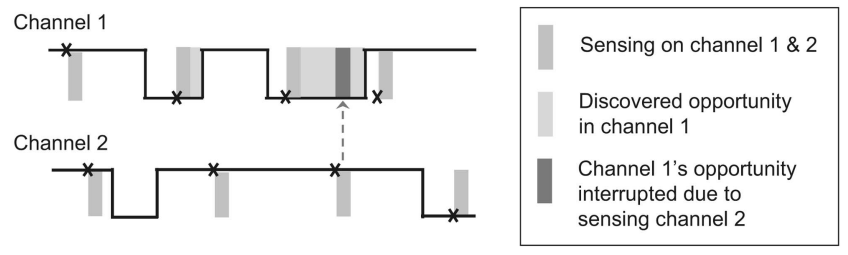

Fig. 5. Concept of $S S O H^{i}$ : Channel 1's discovered opportunity cannot be utilized during sensing of channel 2.

define two mathematical terms, $U O P P^{i}\left(T_{P}^{i}\right)$ (Unexplored Opportunity) and $S_{S O H}\left(T_{P}\right)$ (Sensing Overhead), where $\underline{T_{P}}=\left(T_{P}^{1}, T_{P}^{2}, \ldots, T_{P}^{N}\right) \cdot U O P P^{i}\left(T_{P}^{i}\right)$ is defined as the average fraction of time during which channel $i$ 's opportunities are not discovered in case channel $i$ is being periodically sensed with its sensing period $T_{P}^{i}$. On the other hand, $S S O H^{i}\left(\underline{T_{P}}\right)$ is defined as the average fraction of time during which channel $i$ 's discovered opportunities are interrupted and not utilized due to sensing of one of $N$ channels. An alreadydiscovered opportunity within a channel will be interrupted by sensing because we assumed that 1 ) an $\mathrm{SU}$ is equipped with a single antenna and 2) all SUs in the SN must participate in sensing a channel. That is, the SUs must suspend the use of a discovered channel when it senses other channels since data transmission and sensing cannot take place at the same time with one antenna. This situation is depicted in Fig. 5.

Since $u^{i}$ is defined as the average fraction of time channel $i$ is busy, the average sum of opportunities per unit time is given as $\left(1-u^{i}\right)$. Our objective function is then to find optimal sensing periods of $N$ channels such that

$$
\begin{aligned}
{\underline{T_{P}}}^{*}=\underset{\underline{T_{P}}}{\arg \max }\left\{\begin{array}{c}
\sum_{i=1}^{N}\left\{\left(1-u^{i}\right)-S_{S O H}\left(\underline{T_{P}}\right)\right. \\
\left.-U O P P^{i}\left(T_{P}^{i}\right)\right\}
\end{array}\right\}, \\
=\underset{\underline{T_{P}}}{\arg \min }\left\{\begin{array}{c}
\sum_{i=1}^{N}\left\{S S O H^{i}\left(\underline{T_{P}}\right)\right. \\
\left.+\operatorname{UOPP}^{i}\left(T_{P}^{i}\right)\right\}
\end{array}\right\},
\end{aligned}
$$

where $T_{P}{ }^{*}=\left(T_{P}^{1 *}, \ldots, T_{P}^{N^{*}}\right)$ is a vector of optimal sensing periods. As a boundary condition of $T_{P}^{i}, \sum_{i=1}^{N} \frac{T_{I}^{i}}{T_{P}^{i}}<1$ should be satisfied, providing a lower bound of $T_{P}^{i}$.

\subsection{Analysis of $U O P P^{i}\left(T_{P}^{i}\right)$}

We define $T_{d}^{i}(t)(d=0,1)$ as the average of opportunities (measured in time units) on channel $i$ during $\left(t_{s}, t_{s}+t\right)$, provided a sample $d$ is collected at time $t_{s}$. In case the state transition $(\mathrm{ON} \rightarrow \mathrm{OFF}$ or $\mathrm{OFF} \rightarrow \mathrm{ON})$ occurs at $t_{s}, \tilde{T}_{d}^{i}(t)$ (instead of $T_{d}^{i}(t)$ ) is used to denote the same metric. Possible scenarios of those four functions $T_{0}^{i}(t), T_{1}^{i}(t), \tilde{T}_{0}^{i}(t)$, and $\tilde{T}_{1}^{i}(t)$ are illustrated in Fig. 6. Note that $T_{d}^{i}\left(T_{P}{ }^{i}\right)$ implies the average amount of channel availability between two consecutive samples in case the first sample is $d$.

According to the renewal theory, for an alternating renewal process that has been started a long time ago, the remaining time $\tilde{x}$ in the current state (say, OFF state) from the sampling time $t_{s}$ has its p.d.f. of $\mathbb{F}_{T_{O F F}^{i}}(\tilde{x}) / E\left[T_{O F F}^{i}\right], \tilde{x}>0$

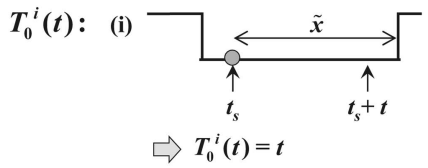

(ii)
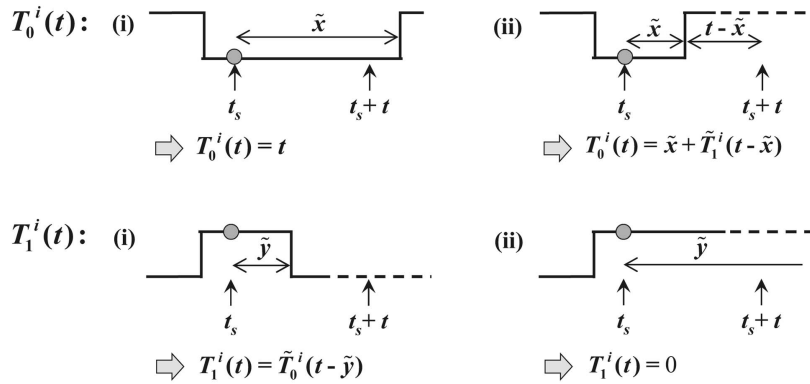

(ii)
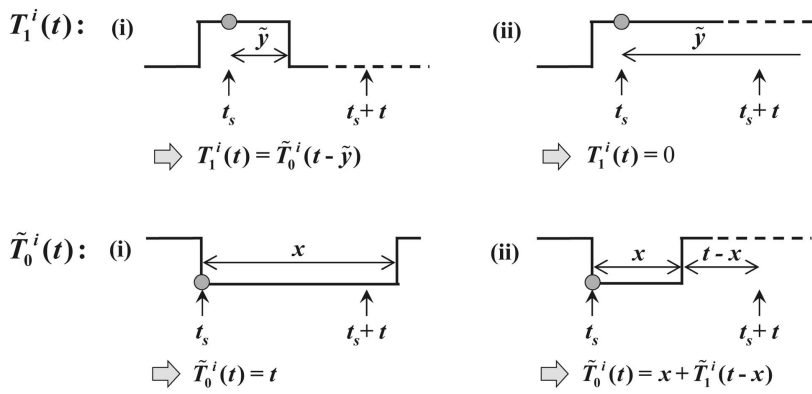

(ii)
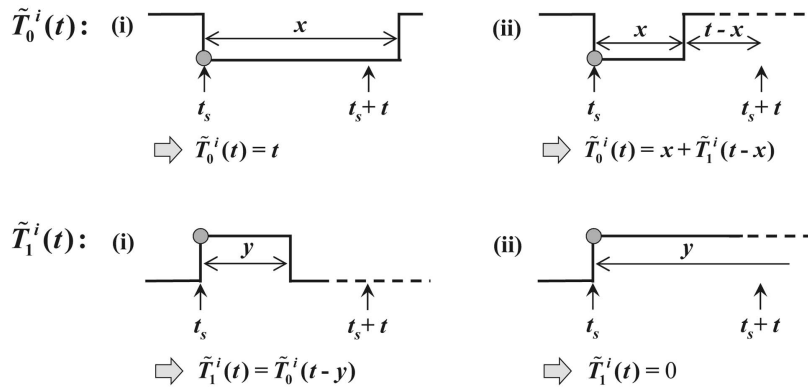

(ii)

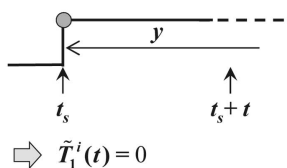

Fig. 6. Illustration of $T_{0}^{i}(t), T_{1}^{i}(t), \tilde{T}_{0}^{i}(t)$, and $\tilde{T}_{1}^{i}(t)$ : For each function, two possible cases 1 and 2 are shown. $\tilde{x} / \tilde{y}$ denotes the remaining time in the current OFF/ON period starting from $t_{s}$. In case the state transition occurs at $t_{s}, x / y$ is used instead of $\tilde{x} / \tilde{y}$.

[20], [21], where $\mathbb{F}_{T_{O F F}^{i}}(\tilde{x})=1-F_{T_{O F F}^{i}}(\tilde{x})$ and $F_{T_{O F F}^{i}}(\tilde{x})$ is the c.d.f. of the OFF period. This is illustrated in Fig. 7, where $\tilde{T}_{O F F}^{i}$ is a random variable of the remaining time in the OFF period. Similarly, the p.d.f. of the remaining time in the $\mathrm{ON}$ state from $t_{s}$ is given as $\mathbb{F}_{T_{O N}^{i}}(\tilde{y}) / E\left[T_{O N}^{i}\right], \tilde{y}>0$. Using the above facts, we can derive the following equations:

$$
\begin{aligned}
T_{0}^{i}(t)= & t \int_{t}^{\infty} \frac{\mathbb{F}_{T_{O F F}^{i}}(x)}{E\left[T_{O F F}^{i}\right] d x} \\
& +\int_{0}^{t} \frac{\mathbb{F}_{T_{O F F}^{i}}(x)}{E\left[T_{O F F}^{i}\right]}\left(x+\tilde{T}_{1}^{i}(t-x)\right) d x, \\
T_{1}^{i}(t)= & \int_{0}^{t} \frac{\mathbb{F}_{T_{O N}^{i}}(y)}{E\left(T_{O N}^{i}\right)} \tilde{T}_{0}^{i}(t-y) d y, \\
\tilde{T}_{0}^{i}(t)= & t \int_{t}^{\infty} f_{T_{O F F}^{i}}(x) d x \\
& +\int_{0}^{t} f_{T_{O F F}^{i}}(x)\left(x+\tilde{T}_{1}^{i}(t-x)\right) d x \\
\tilde{T}_{1}^{i}(t)= & \int_{0}^{t} f_{T_{O N}^{i}}(y) \tilde{T}_{0}^{i}(t-y) d y .
\end{aligned}
$$

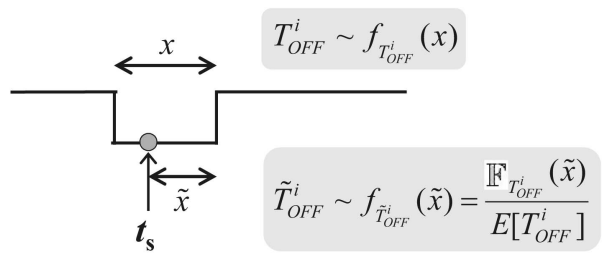

Fig. 7. The density function of the remaining time in the current OFF period. 
By performing the Laplace transform, we get

$$
\begin{aligned}
T_{0}^{i^{*}}(s)= & \left\{\mathbb{F}_{X^{i}}{ }^{*}(0)-\mathbb{F}_{X^{i}}{ }^{*}(s)\right\} /\left\{E\left[T_{O F F}^{i}\right] \cdot s^{2}\right\} \\
& +\mathbb{F}_{X^{i}}{ }^{*}(s) \tilde{T}_{1}^{i *}(s) / E\left[T_{O F F}^{i}\right], \\
T_{1}^{i *}(s)= & \mathbb{F}_{T_{O N}^{i}}{ }^{*}(s) \tilde{T}_{0}^{i *}(s) / E\left[T_{O N}^{i}\right], \\
\tilde{T}_{1}^{i *}(s)= & f_{T_{O N}^{i}}{ }^{*}(s) \tilde{T}_{0}^{i *}(s), \\
\tilde{T}_{0}^{i *}(s)= & \left\{f_{T_{O F F}^{i}}{ }^{*}(0)-f_{T_{O F F}^{i}}{ }^{*}(s)\right\} / s^{2} \\
& +f_{T_{O F F}^{i}}{ }^{*}(s) \tilde{T}_{1}^{i *}(s) .
\end{aligned}
$$

Hence, it leads to

$$
\begin{aligned}
& T_{0}^{i *}(s)=\frac{1}{E\left[T_{O F F}^{i}\right] \cdot s^{2}} \cdot\left[\mathbb{F}_{T_{O F F}^{i}}^{*}(0)-\mathbb{F}_{T_{O F F}^{i}}^{*}(s) .\right. \\
& \left.\frac{1-f_{T_{O F F}^{i}}^{*}(0) f_{T_{O N}^{i}}^{*}(s)}{1-f_{T_{O F F}^{i}}^{*}(s) f_{T_{O N}^{i}}^{*}(s)}\right], \\
& T_{1}^{i *}(s)=\frac{\mathbb{F}_{T_{O N}^{i}}^{*}(s)}{E\left(T_{O N}^{i}\right) \cdot s^{2}} \cdot \frac{f_{T_{O F F}^{i}}^{*}(0)-f_{T_{O F F}^{i}}^{*}(s)}{1-f_{T_{O F F}^{i}}^{*}(s) f_{T_{O N}^{i}}^{*}(s)} .
\end{aligned}
$$

Now, we develop an expression of $U O P P^{i}\left(T_{P}^{i}\right)$ in terms of $T_{0}^{i}(t)$ and $T_{1}^{i}(t) .{ }^{4}$ A new term $U O P P_{(d)}^{i}\left(T_{P}^{i}\right)$ is defined as the average fraction of time during which usable opportunities are not discovered between two consecutive samples in case the first sample is $d$. Then, $U O P P^{i}\left(T_{P}^{i}\right)=$ $\left.\left(1-u^{i}\right) \cdot \operatorname{UOPP}_{(0)}^{i}\left(T_{P}^{i}\right)+u^{i} \cdot \operatorname{UOPP}_{(1)}^{i}\left(T_{P}^{i}\right)\right)^{5}$

In case $d=1$ is collected at time $t_{s}$, opportunities existing in $\left(t_{s}, t_{s}+T_{P}^{i}\right)$ cannot be discovered since there is no more sensing between two sampling times $t_{s}$ and $t_{s}+T_{P}^{i}$. Since the amount of opportunities in $\left(t_{s}, t_{s}+T_{P}^{i}\right)$ is given as $T_{1}^{i}\left(T_{P}{ }^{i}\right)$, we get

$$
\operatorname{UOPP}_{(1)}^{i}\left(T_{P}^{i}\right)=\left[\frac{T_{1}\left(T_{P}^{i}\right)}{T_{P}^{i}}\right] .
$$

In case $d=0$ is collected at time $t_{s}$, the opportunity discovered at $t_{s}$ starts to be utilized until PUs' return. If the OFF period lasts more than $T_{P}^{i}$ after $t_{s}$, there will not be any unexplored portion of opportunities in $\left(t_{s}, t_{s}+T_{P}^{i}\right)$. On the contrary, if PUs emerge at $t_{e}\left(t_{s}<t_{e}<t_{s}+T_{P}^{i}\right)$, any opportunities in $\left(t_{e}, t_{s}+T_{P}^{i}\right)$ could not be explored since the next sampling time is $t_{s}+T_{P}^{i}$. Hence,

$$
\operatorname{UOPP}_{(0)}^{i}\left(T_{P}^{i}\right)=\frac{1}{T_{P}^{i}} \int_{0}^{T_{P}^{i}} \frac{\mathbb{F}_{T_{O F F}^{i}}(x)}{E\left[T_{O F F}^{i}\right]} \tilde{T}_{1}^{i}\left(T_{P}^{i}-x\right) d x,
$$

which completes the derivation of $U O P P^{i}\left(T_{P}^{i}\right)$.

Two examples of $U O P P^{i}\left(T_{P}^{i}\right)$ are introduced here. In case channel $i$ 's ON/OFF periods are Erlang-distributed, we have

$$
\begin{aligned}
f_{T_{O F F}^{i}}(x) & =x e^{-x}, f_{T_{O N}^{i}}(y)=y e^{-y}(x, y>0), \\
\operatorname{UOP}^{i}\left(T_{P}^{i}\right) & =\frac{1}{2}-\frac{3}{4 T_{P}^{i}}+\frac{e^{-T_{P}^{i}}}{4}\left(\frac{3}{T_{P}^{i}}+1\right) .
\end{aligned}
$$

4. Note that $\tilde{T}_{d}^{i}(t)$ can be derived from $T_{d}^{i}(t)$.

5. Note that a channel is assumed to be in its equilibrium state, and in such a case, $u^{i}$ is the probability that a sample 1 is collected from channel $i$ at a random time point [20], [21].

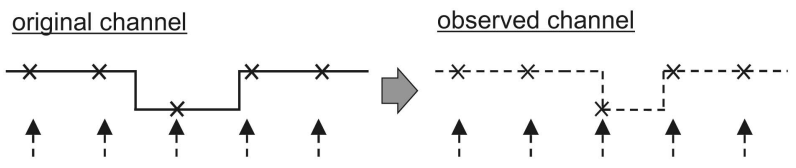

Fig. 8. The observed channel-usage pattern model.

On the other hand, for exponentially distributed ON/OFF periods, we have

$$
\begin{aligned}
& \left\{\begin{array}{l}
f_{T_{O F F}^{i}}(x)=\lambda_{T_{O F F}^{i}} e^{-\lambda_{T_{O F F}^{i}}{ }^{x}}(x>0) \\
f_{T_{O N}^{i}}(y)=\lambda_{T_{O N}^{i}} e^{-\lambda_{T_{O N}^{i}} y}(y>0),
\end{array}\right. \\
& \operatorname{UOPP}^{i}\left(T_{P}^{i}\right)=\left(1-u^{i}\right) \cdot\left\{1-\frac{1-e^{-\lambda_{T_{O F F}^{i}} T_{P}^{i}}}{\lambda_{T_{O F F}^{i}} T_{P}^{i}}\right\} .
\end{aligned}
$$

These results are reasonable in the sense that $\lim _{T_{P}{ }^{i} \rightarrow \infty} U O P P^{i}\left(T_{P}^{i}\right)=1-u^{i}$. As $T_{P}{ }^{i} \rightarrow \infty$, no opportunity is discovered since no sensing will be performed. Therefore, $\operatorname{UOPP}^{i}\left(T_{P}^{i}\right)$ becomes $\left(1-u^{i}\right)$.

\subsection{Analysis of $S S O H^{i}\left(\underline{T_{P}}\right)$}

As defined earlier, $S S O H^{i}\left(T_{P}\right)$ is the average fraction of time during which channel $i$ 's discovered opportunities cannot be utilized due to sensing $N$ channels. To express $S S O H^{i}\left(T_{P}\right)$ mathematically, we introduce a concept of observed channelusage pattern. Since a channel's ON-OFF usage pattern is partially observed by SUs via sensing at discrete-time points, the exact renewal times (that is, state transition times such as $\mathrm{ON} \rightarrow \mathrm{OFF}$ or $\mathrm{OFF} \rightarrow \mathrm{ON}$ ) cannot be observed by SUs. Instead, we use an observed ON-OFF pattern of channel $i$ to derive $S_{S O H}\left(T_{P}\right)$. In the observed ON-OFF model, a channel's OFF period starts when the OFF period is discovered. Once an OFF period is discovered, however, the next state transition to the following ON period is assumed to be recognized via the Listen-before-Talk policy. Fig. 8 illustrates the concept of the new model. This model's channel utilization is called modified channel utilization, denoted by $\tilde{u}^{i}$, which is given as $\tilde{u}^{i}=u^{i}+\operatorname{UOP} P^{i}\left(T_{P}^{i}\right)$.

Using the new model, $\operatorname{SSOH}^{i}\left(\underline{T_{P}}\right)$ can be derived as

$$
S_{S O H}\left(\underline{T_{P}}\right)=\left(1-\tilde{u}^{i}\right) \sum_{j=1}^{N}\left(\frac{T_{I}^{j}}{T_{P}^{j}}\right) .
$$

In the above equation of $S S O H^{i}\left(\underline{T_{P}}\right),\left(1-\tilde{u}^{i}\right)$ implies the time fraction in which channel $i^{\prime}$ s opportunities are discovered. The reason for using $\tilde{u}^{i}$ instead of $u^{i}$ is that $S S O H^{i}$ is only concerned with the discovered portion of OFF periods by its definition. The second term

$$
\sum_{j=1}^{N}\left(\frac{T_{I}^{j}}{T_{P}^{j}}\right)
$$

means the cumulative sensing overhead due to sensing on $N$ channels.

\subsection{Sensing-Period Optimization Algorithm}

Based on the derived expressions of $U O P P^{i}\left(T_{P}^{i}\right)$ and $S S O H^{i}\left(\underline{T_{P}}\right)$, the optimal sensing periods can be determined by solving (1). 


\section{Minimum Channel-Switching Latency Via Optimum Channel-Sequence of Sensing}

We would now like to minimize the time for an SN to find an idle channel when it has to switch channels so that SUs can resume their communication as soon as possible.

\subsection{Analysis}

According to our assumption, SUs must sense $(N-1)$ foreign channels one by one until they can find an idle one. As a simple search-sequence, the channels may be arranged in an ascending order of channel utilizations $\left(u^{i}\right)$, which is not an optimal solution. Instead, we must consider $P_{\text {idle }}^{i}(t)$, the probability that channel $i$ would be idle at a certain time $t$ based on the previous samples. By setting $t$ to the channel-switching triggering time, we can build the optimal sensing sequence as follows:

$$
\left\{\begin{array}{l}
P_{i d l e}^{i}(t)=\operatorname{Pr}\left(Z^{i}(t)=0 \mid \text { all previous samples }\right), \forall i \\
\text { Search channels in descending order of } P_{i}^{i}(t) .
\end{array}\right.
$$

Again, we consider ON-OFF alternating channels. According to the renewal theory, we only need the most recent sample from each channel to derive $P_{\text {idle }}^{i}(t)$. Hence, $P_{i d l e}^{i}(t)$ becomes the transition probability between the most recent sample and its following sample at $t$. Then, $P_{\text {idle }}^{i}(t)=\operatorname{Pr}\left(Z^{i}(t)=0 \mid Z^{i}\left(s^{i}\right)=d^{i}\right)=P_{d^{i} 0}^{i}\left(t-s^{i}\right)$, where $s^{i}$ is the most recent sensing time on channel $i$ and $P_{d^{i} 0}^{i}\left(t-s^{i}\right)$ is the transition probability between two samples $d^{i}$ (at $s^{i}$ ) and $0($ at $t)$. Since $d^{i}=0$ or $1, P_{00}^{i}$ and $P_{10}^{i}$ are considered. The renewal theory suggests that $P_{11}^{i}\left(\Delta^{i}\right), \Delta^{i}=t-s^{i}$, is expressed as

$$
\begin{aligned}
P_{11}^{i}\left(\Delta^{i}\right)= & \int_{\Delta^{i}}^{\infty} \frac{\mathbb{F}_{T_{O N}^{i}}(u)}{E\left[T_{O N}^{i}\right]} d u \\
& +\int_{0}^{\Delta^{i}} h_{10}^{i}(u) \mathbb{F}_{T_{O N}^{i}}\left(\Delta^{i}-u\right) d u,
\end{aligned}
$$

where $h_{10}^{i}(u)$ is the renewal density of the OFF state given that the renewal process started from the ON state. It is proven in [20] that $h_{10}^{i *}(s)$ is expressed as

$$
h_{10}^{i *}(s)=\frac{f_{T_{O F F}^{i}}^{*}(s)\left\{1-f_{T_{O N}^{i}}^{*}(s)\right\}}{E\left[T_{O N}^{i}\right] \cdot s\left\{1-f_{T_{O N}^{i}}^{*}(s) f_{T_{O F F}^{i}}^{*}(s)\right\}} .
$$

By applying the Laplace transform, we get

$$
P_{11}^{i *}(s)=\frac{1}{s}-\frac{\left\{1-f_{T_{O N}^{i}}^{*}(s)\right\}\left\{1-f_{T_{O F F}^{i}}^{*}(s)\right\}}{E\left[T_{O N}^{i}\right] \cdot s^{2}\left\{1-f_{T_{O N}^{i}}^{*}(s) f_{T_{O F F}^{i}}^{*}(s)\right\}} .
$$

$P_{10}^{i}\left(\Delta^{i}\right)$ can be derived by the inverse Laplace transform along with the following relationship: $P_{10}^{i}\left(\Delta^{i}\right)=1-P_{11}^{i}\left(\Delta^{i}\right)$. By switching the role of state ON and OFF, $P_{00}^{i}\left(\Delta^{i}\right)$ can be easily derived by the inverse Laplace transform of the following term:

$$
P_{00}^{i *}(s)=\frac{1}{s}-\frac{\left\{1-f_{T_{O F F}^{i}}^{*}(s)\right\}\left\{1-f_{T_{O N}^{i}}^{*}(s)\right\}}{E\left[T_{O F F}^{i}\right] \cdot s^{2}\left\{1-f_{T_{O F F}^{i}}^{*}(s) f_{T_{O N}^{i}}^{*}(s)\right\}} .
$$

For example, for a channel with Erlang-distributed ON/ OFF periods, as shown in (2), we have

$$
\begin{aligned}
& P_{00}^{i}\left(\Delta^{i}\right)=\frac{1}{2}+\frac{1}{2} e^{-\Delta^{i}} \cos \left(\Delta^{i}\right), \\
& P_{10}^{i}\left(\Delta^{i}\right)=\frac{1}{2}-\frac{1}{2} e^{-\Delta^{i}} \cos \left(\Delta^{i}\right) .
\end{aligned}
$$

On the other hand, for a channel with exponentially distributed ON/OFF periods as shown in (3), we get

$$
\begin{aligned}
& P_{00}^{i}\left(\Delta^{i}\right)=\left(1-u^{i}\right)+u^{i} \cdot e^{-\left(\lambda_{T_{O F F}^{i}}+\lambda_{T_{O N}^{i}}\right) \Delta^{i}}, \\
& P_{10}^{i}\left(\Delta^{i}\right)=\left(1-u^{i}\right)-\left(1-u^{i}\right) \cdot e^{-\left(\lambda_{T_{O F}^{i}}+\lambda_{T_{O N}^{i}}\right) \Delta^{i}} .
\end{aligned}
$$

\subsection{Optimal Channel-Sequencing Algorithm}

The complete optimal channel-sequencing algorithm is given below.

1. $\forall i$, except that the channel to switch from

$$
\begin{gathered}
\text { calculate } P_{i d l e}^{i}\left(\Delta^{i}\right)= \begin{cases}P_{00}^{i}\left(\Delta^{i}\right), \text { if } d^{i}=0 \\
P_{10}^{i}\left(\Delta^{i}\right), \text { if } d^{i}=1,\end{cases} \\
\text { where } \begin{cases}d^{i}: & \text { most recent sample of channel } i \\
\Delta^{i}: & \text { elapsed amount of time since } \\
& \text { the most recent sensing until } \\
\text { channel-switching. }\end{cases}
\end{gathered}
$$

2. Optimal sensing order. Sense $(N-1)$ channels in descending order of $P_{i d l e}^{i}\left(\Delta^{i}\right)$.

In case one round of channel search for all $(N-1)$ channels cannot find any idle channel, an instant replay of the optimal channel searching is unlikely to find an idle channel because two consecutive samples collected within a short time window on one channel have non-negligible correlation, as will be shown in (4) in Section 6. Therefore, we recommend $(N-1)$ channels to be searched again after $T_{\text {retry }}$ seconds, which is a design parameter of the algorithm. In such a case, a new idle channel will be found by the research of the channels or by regular periodic sensing. In either case, once an idle channel is found, the channelswitching procedure completes and SUs resume their communication on the new channel.

\subsection{Discussion}

Sometimes it is necessary to classify sensing time $T_{I}^{i}$ into two categories: 1) a longer sensing time before SUs resume their communication on a new channel and 2) a shorter sensing time to detect PUs' return on the channel currently being utilized by SUs. In IEEE 802.22, for example, Channel Availability Check Time is defined as the time during which a TV channel shall be checked for the presence of licensed incumbent signals prior to the commencement of WRAN operation in that channel [22], [23]. This parameter corresponds to the first category. On the other hand, a channel which is being utilized by SUs in 802.22 is monitored by sensors by scheduling fast and fine sensing, which requires a much shorter time (less than a few dozen milliseconds) and, hence, corresponds to the second category. 
The proposed analysis can be extended to have two types of sensing time: $T_{I, l o n g}^{i}$ and $T_{I, \text { short }}^{i} . T_{I, l o n g}^{i}$ can be used at channel switching in which each foreign channel should be monitored for a longer time to protect licensed users' operation on the channel. On the other hand, $T_{I, \text { short }}^{i}$ can be used for collecting binary samples to estimate ON/OFF usage patterns.

\section{Channel-Parameter Estimation}

As discussed in Section 3, the estimation of the underlying channel-usage patterns is important to the proposed approach. With an ON-OFF alternating renewal channel, the distribution parameters of ON/OFF periods are to be estimated.

\subsection{Maximum Likelihood (ML) Estimators}

Suppose we have a vector of $r^{i}$ samples from channel $i$, $\underline{Z}^{i}=\left(Z_{t_{1}}^{i}, Z_{t_{2}}^{i}, \ldots, Z_{t_{r_{i}}}^{i}\right)$, where $t_{j}\left(j=1, \ldots, r^{i}\right)$ denotes the time stamp of sample $Z_{t_{j}}^{i}$. Suppose the density functions of $\mathrm{ON}$ and OFF periods are $m$-variate, then a total of $2 m$ parameters should be estimated. On the other hand, the joint probability mass function of $r^{i}$ samples can be expressed with four types of transition probabilities $(0 \rightarrow 0,0 \rightarrow 1,1 \rightarrow 0$, and $1 \rightarrow 1)$ as follows:

$$
\begin{aligned}
\underline{\theta}^{i}= & \left(\theta_{1}^{i}, \ldots, \theta_{2 m}^{i}\right), \\
L\left(\underline{\theta}^{i}\right)= & P\left(\underline{Z}^{i} ; \underline{\theta}^{i}\right) \\
= & \operatorname{Pr}\left(Z_{t_{1}}^{i}=z_{1} ; \underline{\theta}^{i}\right) . \\
& \prod_{k=2}^{r^{i}} \operatorname{Pr}\left(Z_{t_{k}}^{i}=z_{k} \mid Z_{t_{k-1}}^{i}=z_{k-1} ; \underline{\theta}^{i}\right) \\
= & \operatorname{Pr}\left(Z_{t_{1}}^{i}=z_{1} ; \underline{\theta}^{i}\right) \prod_{k=2}^{r^{i}} P_{z_{k-1} z_{k}}^{i}\left(t_{k}-t_{k-1} ; \underline{\theta}^{i}\right),
\end{aligned}
$$

where the Markovian property has been applied. $P_{z_{k-1} z_{k}}^{i}\left(t_{k}-\right.$ $t_{k-1}$ ) denotes the probability that a sample $z_{k-1}$ is followed by a sample $z_{k}$ and the intersample collection time is $t_{k}-t_{k-1}$. Then, the estimates of parameters of ON/OFF density functions can be found by ML estimation, such as

$$
\frac{\partial \ln L\left(\underline{\theta}^{i}\right)}{\partial \theta_{l}^{i}}=0, l=1, \ldots, 2 m .
$$

Now, the remaining task is to express the likelihood function in a mathematical form. The first component of the likelihood function is given as $\operatorname{Pr}\left(Z_{t_{1}}^{i}=z_{1} ; \underline{\theta}^{i}\right)=$ $\left(u^{i}\right)^{z_{1}}\left(1-u^{i}\right)^{1-z_{1}}$ since $u^{i}$ is the probability that channel $i$ is busy (that is, $\mathrm{ON}$ ) at a random time ( $t_{1}$ in this case). Note that the estimator of $u^{i}$ is simply given as the sample mean of $r^{i}$ samples. Another part of the likelihood function is $P_{z_{k-1} z_{k}}^{i}\left(t_{k}-t_{k-1} ; \underline{\theta}^{i}\right)$, which is one of four transition probabilities: $P_{00}^{i}\left(t_{k}-t_{k-1}\right), P_{01}^{i}\left(t_{k}-t_{k-1}\right), P_{10}^{i}\left(t_{k}-t_{k-1}\right)$, and $P_{11}^{i}\left(t_{k}-t_{k-1}\right)$. The derivation of those four functions has already been introduced in Section 5 .

For example, for a channel with exponentially distributed ON/OFF periods as shown in (3), transition probabilities are given as

$$
\begin{aligned}
& P_{00}^{i}(t)=\left(1-u^{i}\right)+u^{i} \cdot e^{-\left(\lambda_{T_{O F F}^{i}}+\lambda_{T_{O N}^{i}}\right) t}, \\
& P_{01}^{i}(t)=u^{i}-u^{i} \cdot e^{-\left(\lambda_{T_{O F F}^{i}}+\lambda_{T_{O N}^{i}}\right) t}, \\
& P_{11}^{i}(t)=u^{i}+\left(1-u^{i}\right) \cdot e^{-\left(\lambda_{T_{O F F}^{i}}+\lambda_{T_{O N}^{i}}\right) t}, \\
& P_{10}^{i}(t)=\left(1-u^{i}\right)-\left(1-u^{i}\right) \cdot e^{-\left(\lambda_{T_{O F F}^{i}}+\lambda_{T_{O N}^{i}}\right) t} .
\end{aligned}
$$

Then, there are two parameters to be estimated: $\lambda_{T_{O F F}^{i}}$ and $\lambda_{T_{O N}^{i}}$. Since

$$
u^{i}=\frac{E\left[T_{O N}^{i}\right]}{E\left[T_{O N}^{i}\right]+E\left[T_{O F F}^{i}\right]}=\frac{\lambda_{T_{O F F}^{i}}}{\lambda_{T_{O N}^{i}}+\lambda_{T_{O F F}^{i}}},
$$

we can estimate $\lambda_{T_{O F F}^{i}}$ and $u^{i}$ instead of $\lambda_{T_{O F F}^{i}}$ and $\lambda_{T_{O N}^{i}}$.

As already discussed, the estimator of $u^{i}$ is given as

$$
\hat{u}^{i}=\frac{1}{r^{i}} \sum_{k=1}^{r^{i}} Z_{t_{k}}^{i}
$$

On the other hand, the estimator of $\lambda_{T_{O F F}^{i}}$ can be derived by solving the equation $\partial \ln L(\underline{\theta}) / \partial \lambda_{T_{O F F}^{i}}=0$, yielding

$$
\begin{aligned}
& \hat{\lambda}_{T_{\text {OFF }}^{i}}=-\frac{u^{i}}{T_{P}^{i}} \ln \left[\frac{-B+\sqrt{B^{2}-4 A C}}{2 A}\right], \\
& \text { where }\left\{\begin{array}{l}
A=\left(u^{i}-\left(u^{i}\right)^{2}\right)\left(r^{i}-1\right), \\
B=-2 A+\left(r^{i}-1\right)-\left(1-u^{i}\right) n_{0}-u^{i} \cdot n_{3}, \\
C=A-u^{i} \cdot n_{0}-\left(1-u^{i}\right) n_{3} .
\end{array}\right.
\end{aligned}
$$

Note that $n_{0} / n_{1} / n_{2} / n_{3}$ indicates the number of $(0 \rightarrow$ $0) /(0 \rightarrow 1) /(1 \rightarrow 0) /(1 \rightarrow 1)$ transitions from the total of $\left(r^{i}-1\right)$ transitions among $r^{i}$ samples. For instance, in case a sequence of samples is given as $(0,1,1,1,0,1,1,0), r^{i}=8$, we have $n_{0}=0, n_{1}=2, n_{2}=2$, and $n_{3}=3$.

\subsection{Confidence Interval of Estimators}

It is also important to understand how much one can have confidence in the derived estimators. The confidence interval is an efficient measure to determine the level of confidence. In most cases, however, it is not easy or sometimes impossible to derive the confidence interval in a closed form with generally formed density functions of $\mathrm{ON} / \mathrm{OFF}$ periods. Here, we show the derivation of confidence intervals with exponentially distributed $\mathrm{ON} /$ OFF periods.

\subsubsection{Confidence Interval of $\hat{u}^{i}$}

When channel $i$ is periodically sensed at an interval $T_{P}^{i}$, the difference between any two time stamps $Z_{t_{k_{1}}}$ and $Z_{t_{k_{2}}}$ $\left(k_{1}, k_{2} \in\left\{1,2, \ldots, r^{i}\right\}\right)$ is an integer multiple of $T_{P}^{i}$. In such a case, the correlation coefficient of any two samples $Z_{t_{k_{1}}}$ and $Z_{t_{k_{2}}}\left(k_{1}>k_{2}\right)$ is found to be

$$
\begin{aligned}
E\left[Z_{t_{k_{1}}} Z_{t_{k_{2}}}\right] & =\operatorname{Pr}\left(Z_{t_{k_{1}}}=1 \mid Z_{t_{k_{2}}}=1\right) \operatorname{Pr}\left(Z_{t_{k_{2}}}=1\right) \\
& =P_{11}^{i}\left(\left|k_{1}-k_{2}\right| \cdot T_{P}^{i}\right) \cdot u^{i}, \\
\Rightarrow \rho_{k_{1} k_{2}} & =\frac{E\left[Z_{t_{k_{1}}} Z_{t_{k_{2}}}\right]-\left(u^{i}\right)^{2}}{u^{i}-\left(u^{i}\right)^{2}} \\
& =e^{-\left(\lambda_{T_{O F F}^{i}} / u^{i}\right) \cdot\left|k_{1}-k_{2}\right| T_{P}^{i}} .
\end{aligned}
$$




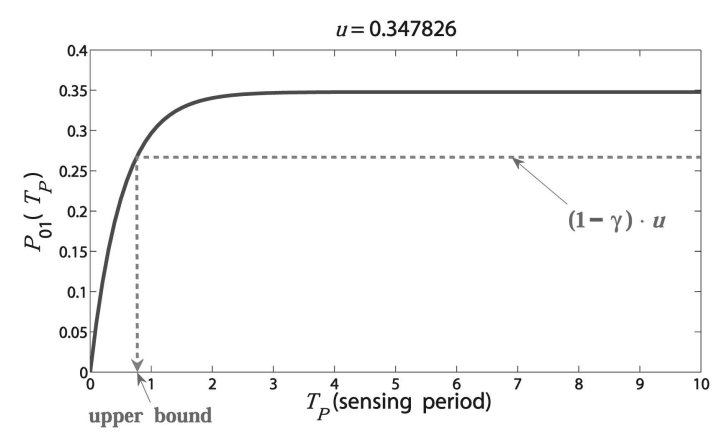

Fig. 9. The graph of $P_{01}^{i}\left(T_{P}^{i}\right)$ and upper bound of $T_{P}^{i}$.

This shows that the correlation is decaying fast (exponentially) as the separation of two sampling times becomes large. Since the rate of decrease is proportional to $\left(\lambda_{T_{O F F}^{i}} / u^{i}\right) T_{P}^{i}, r^{i}$ samples can be assumed to be weakly correlated, as $r^{i}$ are large unless $\left(\lambda_{T_{O F F}^{i}} / u^{i}\right) T_{P}^{i}$ is close to 0 . Using this fact, we can derive the confidence interval. When $\left(\lambda_{T_{O F F}^{i}} / u^{i}\right) T_{P}^{i}$ is not close to $0, \frac{\overline{Z^{i}}-E\left[\overline{Z^{i}}\right]}{\left.\sqrt{\operatorname{var}\left[Z^{i}\right.}\right]} \rightarrow N(0,1)$ as $r^{i} \rightarrow \infty$ by the Central Limit Theorem. ${ }^{6}$ Hence, $100(1-\alpha)$ percent confidence interval is given as

$$
\begin{aligned}
{\left[\overline{Z^{i}}-\sqrt{\operatorname{var}\left[\overline{Z^{i}}\right]} \cdot N^{-1}(1-\alpha / 2),\right.} \\
\left.\overline{Z^{i}}+\sqrt{\operatorname{var}\left[\overline{Z^{i}}\right]} \cdot N^{-1}(1-\alpha / 2)\right] .
\end{aligned}
$$

where $\operatorname{var}\left[\overline{Z^{i}}\right]$ is a function of $r^{i}$. If $\beta \equiv \sqrt{\operatorname{var}\left[\overline{Z^{i}}\right]}$. $N^{-1}(1-\alpha / 2), r^{i}$ can be related to the level $\alpha$ of confidence with the interval length of $2 \beta$. In general, we need more samples (that is, bigger $r^{i}$ ) to achieve a higher level of confidence (that is, smaller $\alpha$ or $\beta$ ).

\subsubsection{Confidence Interval of $\hat{\lambda}_{T_{O F F}^{i}}$}

The ML estimator of $\lambda_{T_{O F F}^{i}}$ has already been shown. Unfortunately, the high nonlinearity of $\hat{\lambda}_{T_{O F F}^{i}}$ makes it difficult to find its exact confidence interval. Instead, an upper bound of $T_{P}^{i}$ could be derived to ensure a reasonable level of confidence. Note that each of four transition probabilities tends to converge to a constant $\left(u^{i}\right.$ or $\left.1-u^{i}\right)$, as $T_{P}^{i}$ goes to infinity. Since $\ln L\left(\underline{\theta}^{i}\right)$ is expressed with transition probabilities, an ML estimator cannot guarantee accurate estimation with a large $T_{P}^{i}$ with which the likelihood function tends to be a constant. Hence, we will bound the value of $P_{01}^{i}\left(T_{P}^{i}\right)$ below a certain threshold (1r) $u^{i}$ to ensure that the probability would not be too close to its limit. This concept is shown in Fig. 9. Then, an upper bound of $T_{P}^{i}$ can be derived as

$$
\left|u^{i}-P_{01}^{i}\left(T_{P}^{i}\right)\right| \geq \gamma \times u^{i} \Rightarrow T_{P}^{i} \leq \frac{u^{i}}{\lambda_{T_{O F F}^{i}}} \ln \left(\frac{1}{\gamma}\right) .
$$

6. $\overline{Z^{i}}$ is the sample mean of $\underline{Z}^{i}=\left(Z_{t_{1}}^{i}, Z_{t_{2}}^{i}, \ldots, Z_{t_{r_{i}}}^{i}\right)$.
Hence, the optimal sensing period in Section 4 should be determined subject to the constraint of the upper bound of $T_{P}^{i}$ given here.

\subsubsection{Discussion}

We can apply the same intuition derived from the case of exponentially distributed ON/OFF periods to general distributions. First, upon the estimation of channel utilization $u^{i}$, the more samples are given, the more accurate the estimates. On the other hand, if we want to estimate $E\left[T_{O F F}^{i}\right]$ and $E\left[T_{O N}^{i}\right]$, it is important to upper bound $T_{P}^{i}$ so that a sufficient number of samples would be collected within each $\mathrm{OFF} / \mathrm{ON}$ period. If $T_{P}^{i}$ increases, both $E\left[T_{O F F}^{i}\right]$ and $E\left[T_{O N}^{i}\right]$ will be overestimated, as many OFF/ON periods would be missed by the sensing. Therefore, the number of samples and sensing frequency are two important factors that control the confidence level of estimation.

\subsection{Estimation on Time-Varying Channel}

The ON-OFF patterns of licensed channels are often time varying, implying that the parameter estimation must be adaptive in time. Here, we assume that the channel parameters of ON/OFF periods are slowly time varying so that the SUs can track their variations by using a moving time window in collecting samples and making estimations. That is, channel $i$ 's sensing results (binary samples) are preserved for those whose sampling time stamps are no older than $T_{\text {window }}^{i}$, where $T_{\text {window }}^{i}$ indicates the time-window size of channel $i$. The estimation procedure must be executed frequently enough to track the variation of parameters. As an extreme case, new estimates might be produced every time when a new sample is collected from a channel, although it may incur high processing cost. Therefore, in Section 7, we compute estimates once every $T_{\text {estimation }}$ seconds, which is much smaller than $T_{\text {window }}^{i}$.

Whenever new estimates are computed, the optimal sensing periods derived in Section 4 must be recalculated and adapted accordingly. The optimal sensing sequence at channel switching, however, can be reactively derived at every channel switch.

\section{Evaluation}

\subsection{Simulation Setup}

To measure the effectiveness of the proposed schemes, we define two performance metrics: Achieved Opportunity Ratio (AOR) and Channel Switching Latency (CSL).

AOR measures the ratio of the total discovered spectrum availability to the total existing availability. This metric will show the efficiency of the proposed sensing period optimization in terms of the percentage of total opportunities it can discover. Ideally, if all estimates are perfect, AOR will be as high as

$$
A O R_{\max }=\frac{\sum_{i=1}^{N}\left\{\begin{array}{c}
\left(1-u^{i}\right)-S S O H^{i}\left(T_{P}^{*}\right) \\
-U O P P^{i}\left(\overline{T_{P}^{i *}}\right)
\end{array}\right\}}{\sum_{i=1}^{N}\left(1-u^{i}\right)},
$$


This article has been accepted for inclusion in a future issue of this journal. Content is final as presented, with the exception of pagination.

TABLE 1

General Evaluation Parameters

\begin{tabular}{|c|c|c|c|}
\hline$\gamma$ & $T_{\text {window }}^{i}(\forall i)$ & $T_{\text {estimation }}$ & $T_{I}^{i}(\forall i)$ \\
\hline 0.2 & $200(\mathrm{sec})$ & $20(\mathrm{sec})$ & $2(\mathrm{~ms})$ \\
\hline
\end{tabular}

where the numerator comes from (1). In practice, however, $A O R_{\max }$ cannot be achieved since estimates are not perfect. We will show how much the actual simulation results deviate from $A O R_{\max }$.

CSL is defined as the average time (in seconds) taken to find an idle channel upon triggering a channel switch. It will be used to assess the efficiency of the proposed optimal sensing-sequencing algorithm.

In addition to the above two metrics, we also study how close the estimation results would be to the actual channel parameters and how well estimates track time-varying channels.

We conducted simulation using Matlab, and all $N$ channels are assumed to have exponentially distributed ON/ OFF periods. A total of nine heterogeneous channels have been used $(i=1,2, \ldots, 9)$, where $\left(\lambda_{T_{O F F}}^{i}, \lambda_{T_{O N}}^{i}\right)$ are independently chosen. We tested different channel conditions by changing the number of channels to be sensed such as 1) three channels (channel 1,2,3), 2) six channels (channel $1,2, \ldots, 6)$, and 3) nine channels (channel $1,2, \ldots, 9)$. For each case, a simulation ran for 5,000 seconds and AOR/CSL were measured. To observe the average behavior, the simulation under the same condition was repeated 10 times.

To emulate time-varying channel conditions, the channel parameter $\lambda_{T_{O F F}}^{i} / \lambda_{T_{O N}}^{i}$ decreases/increases its value by 10 percent once every 1,000 seconds. This allows us to show the efficiency of parameter tracking of our estimation with a moving time window.

The proposed scheme is comparatively evaluated against other schemes. For the AOR test, our scheme with sensingperiod optimization is compared to the reference scheme without sensing-period optimization. For both schemes, optimal sensing sequencing is turned off. Since there is no sensing-period adaptation in the reference scheme, it starts with a randomly chosen initial sensing period, which will not be adapted. The reference scheme is tested with four different initial $T_{P}^{i}$ : $0.05,0.1,0.5$, and 1.0 seconds $(\forall i)$. For the proposed scheme, the algorithm starts with the initial $T_{P}^{i}$ of 0.5 (seconds) and is then adapted and optimized gradually. This comparison will clearly show the importance of sensing-period optimization to efficient collection of more opportunities.

TABLE 2

Test-Specific Evaluation Parameters

\begin{tabular}{|c|c|c|}
\hline [1] AOR test & Proposed & Reference \\
\hline Initial $T_{P}^{i}$ & $0.5(\mathrm{sec})$ & $0.05 / 0.1 / 0.5 / 1.0(\mathrm{sec})$ \\
\hline \hline [2] CSL test & \multicolumn{2}{|c|}{ Proposed \& Compared } \\
\hline Initial $T_{P}^{i}$ & \multicolumn{2}{|c|}{$0.5(\mathrm{sec})$} \\
$T_{\text {retry }}$ & \multicolumn{2}{|c|}{$0.05 / 0.1 / 0.5(\mathrm{sec})$} \\
\hline
\end{tabular}

TABLE 3

Channel-Usage Pattern Parameters

\begin{tabular}{|c|c|c|c|c|c|}
\hline & Ch 1 & Ch 2 & Ch 3 & Ch 4 & Ch 5 \\
\hline$E\left[T_{O F F}^{i}\right]$ & 1.50 & 0.50 & 1.00 & 3.00 & 1.00 \\
$E\left[T_{O N}^{i}\right]$ & 0.80 & 2.50 & 1.00 & 2.50 & 2.00 \\
\hline & Ch 6 & Ch 7 & Ch 8 & Ch 9 & \\
\hline$E\left[T_{O F F}^{i}\right]$ & 3.50 & 4.00 & 0.50 & 0.75 & \\
$E\left[T_{O N}^{i}\right]$ & 0.50 & 1.00 & 5.50 & 2.00 & \\
\hline
\end{tabular}

On the other hand, for the CSL test, the proposed scheme with sensing-period optimization and optimal channel-sequencing will be compared with another scheme with sensing-period optimization but without optimal channel sequencing. The latter scheme is called the compared scheme. In the compared scheme, a new channel is discovered only by periodic sensing and there is no ondemand sensing to discover an idle channel when the channel needs to be switched. In both cases, CSL is measured and compared. This helps us evaluate the benefit of optimal channel sequencing in minimizing the delay of regaining an idle channel when the channel needs to be switched. We also study the effect of $T_{\text {retry }}$ on CSL by trying three different values of the design parameter $T_{\text {retry }}$ : $0.05,0.1$, and 0.5 seconds.

The parameters used for the simulation are shown in Tables 1, 2 and 3, where $E\left[T_{O F F}^{i}\right]$ and $E\left[T_{O N}^{i}\right]$ are in seconds.

\subsection{The Simulation Results}

\subsubsection{Achieved Opportunity Ratio (AOR)}

Fig. 10 plots the AOR of the proposed and reference schemes. The x-axis represents the number $N$ of licensed channels and $y$-axis presents AOR in percent. One hundred percent indicates that a scheme can discover/utilize all existing opportunities of $\sum_{i=1}^{N}\left(1-u^{i}\right)$, which is impossible to achieve in practice due to the sensing overhead $\left(S S O H^{i}\right)$ and the missed portion of opportunities $\left(U O P P^{i}\right)$. Thus, it is more meaningful to consider the analytical maximum of utilizable opportunities $\left(A O R_{\max }\right)$.

The results in the figure show the superiority of the proposed algorithm. The sensing-period optimization offers more than 98 percent of the analytical maximum of discovered spectrum availability regardless of the tested conditions $(N=3,6$, or 9). The small deviation of the performance from $A O R_{\max }$ comes from three factors: 1) the time for estimates to converge to the time-varying

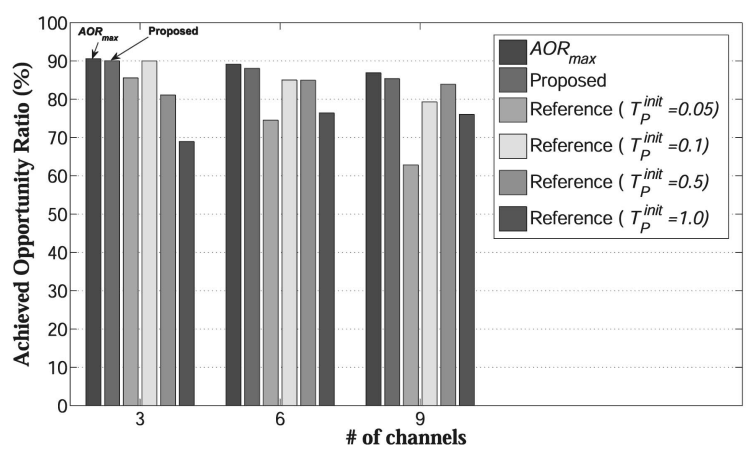

Fig. 10. AOR. 


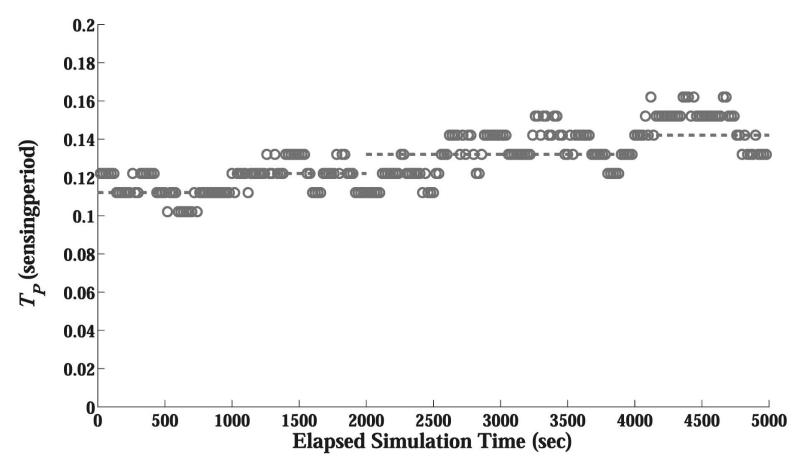

Fig. 11. Adaptation of sensing periods $(\mathrm{N}=3$ case: channel 1$)$.

parameters, 2) the time for sensing periods to be adapted to optimal ones, and 3) small deviation of estimates from the actual parameters. It also discovers up to 22 percent more opportunities than the reference schemes, which do not offer sensing-period optimization. This improvement may become greater as the initial $T_{P}^{i}$ is chosen smaller than 0.05 seconds or larger than 1.0 seconds. In fact, no reference scheme can outperform the proposed scheme. As the initial $T_{P}^{i}$ is chosen farther away from the optimal one, the performance of the reference scheme degrades greatly for two reasons: First, if $T_{P}^{i}$ grows, $S S O H^{i}$ gets smaller but $U O P P^{i}$ becomes larger and dominant, resulting in many missed OFF periods. In contrast, if $T_{P}^{i}$ decreases, $U O P P^{i}$ gets smaller and more OFF periods are discovered, but $S S O H^{i}$ becomes larger, resulting in frequent interrupts in utilizing discovered opportunities on a channel due to sensing channels. Hence, in either case, the reference scheme cannot reach $A O R_{\max }$. One may claim that, in some cases (for example, initial $T_{P}^{i}=0.1$ at $N=3$ ), the reference scheme nearly achieved $A O R_{\max }$. The reference scheme, however, chooses an initial $T_{P}^{i}$ randomly and does not optimize it. Therefore, it would be just pure luck if the reference scheme chooses its initial $T_{P}^{i}$ close to the optimal one. In addition, nonadaptive $T_{P}^{i}$ cannot track time-varying channel environments and will eventually yield poor performance if the simulation was run long enough. On the other hand, the sensing periods in the proposed scheme are adapted to the optimal values in a few cycles, and they also track changing optimal values, as shown in Fig. 11, where adaptation is performed every

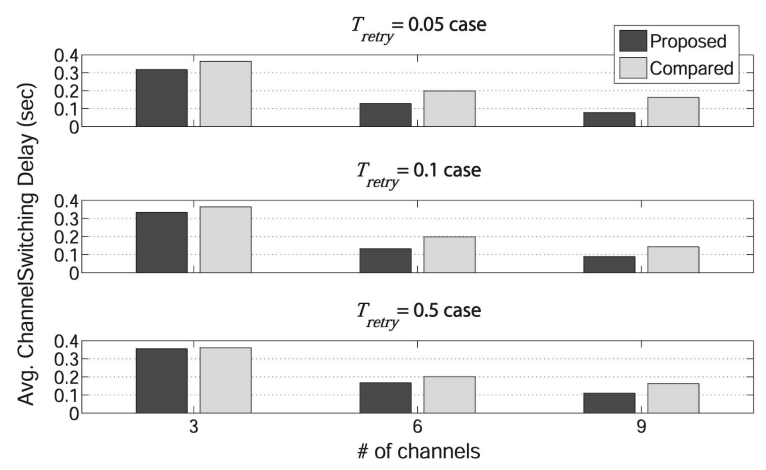

Fig. 12. Channel-switching latency.

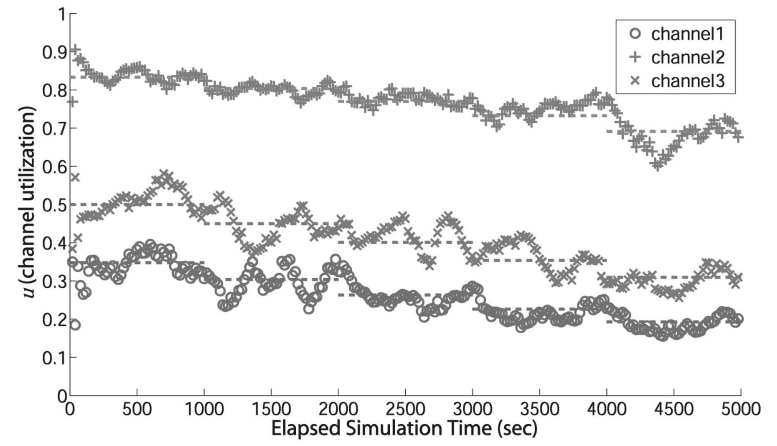

Fig. 13. Estimation of $u^{i}: \mathrm{N}=3$ case.

$T_{\text {estimation }}(\mathrm{sec})$. Dashed lines indicate analytically derived target optimal sensing periods.

\subsubsection{Channel-Switching Latency (CSL)}

With respect to CSL, our scheme also outperforms the compared scheme, which does not perform optimal channel sequencing, as shown in Fig. 12. The delay spans from 0.08 to 0.35 second in our proposed scheme and becomes smaller as the number of channels $(N)$ grows (with fixed $T_{\text {retry }}$ ), mainly because there is a higher chance to find an idle channel with more channels.

For different $T_{\text {retry }}$ values (with fixed $N$ ), the compared scheme's performance shows no significant difference since it does not perform any on-demand search at channel switching nor any re-search with $T_{\text {retry }}$. In contrast, the proposed scheme's CSL increases slightly with $T_{\text {retry }}$ since $(N-1)$ channels would be re-searched less frequently. Although decreasing $T_{\text {retry }}$ could result in a smaller delay in locating a new idle channel, frequent retries may not improve CSL significantly because the channels being rechecked might still be in their ON periods. Therefore, for an $\mathrm{SN}$ with energy constraints, finding a proper $T_{\text {retry }}$ would be an important factor to consider.

\subsubsection{Channel-Parameter Estimation Accuracy}

Figs. 13 and 14 show the accuracy of our channel-usage pattern estimation. Each point in the figure indicates the estimate produced within an estimation cycle, $T_{\text {estimation }}$. Dashed lines represent the actual target channel parameters. The plot of $\hat{u}^{i}$, as well as $\hat{\lambda}_{T_{O F F}^{i}}$, follows the actual channel parameters very closely even when they are time

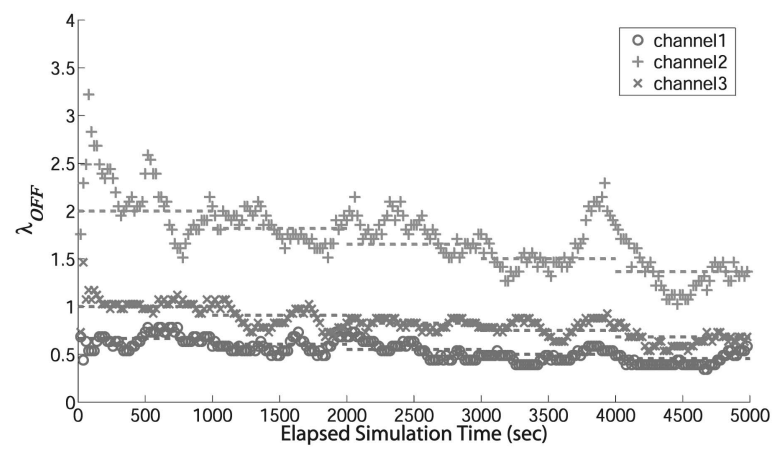

Fig. 14. Estimation of $\lambda_{O F F}^{i}: \mathrm{N}=3$ case. 
varying. The difference between estimates and target values can be controlled by adapting $\gamma$ and $T_{w i n d o w}^{i}$, which are related to the number of samples and confidence intervals of estimators.

\section{Deployment Scenarios}

This section discusses how our proposed schemes can be applied to IEEE 802.11 systems, as well as other licensed bands.

\subsection{Application to IEEE $\mathbf{8 0 2 . 1 1}$}

First, due to limitations in CSMA, 802.11 cannot be directly used as a platform of the proposed schemes. The Clear Channel Assessment (CCA) in CSMA uses energy detection to determine if there is any signal activity in each time slot and, hence, cannot differentiate SU signals from PU signals. As a result, all SUs could be backed off in case there is a PU signal within a contention period since every SU may think the channel is occupied by another SU, not by PUs. It is, however, not desirable because all SUs have to vacate the channel if there exists any PU.

Hence, the current CCA of 802.11 should be modified in such a way that, during a quiet period, no random back-off or data transmission is performed. In addition, the measurement result (busy or idle) of every slot during the quiet period must be reported to the MAC layer and the MAC should also be modified so that the sensing results can be distributed to and shared by SUs.

To support periodic sensing scheduling on home and foreign channels, 802.11 MAC should introduce a new feature to reserve quiet periods. It can be done by using beacons or regular packets exchanged among SUs.

One example of using the 802.11 platform/protocol to implement sensing functionalities is described in our recent paper [15], where we have implemented an incumbent detection mechanism using a commercial WLAN device (Atheros) and its open source device driver, MadWiFi. In terms of sensing periods, a home channel is regularly monitored once every frame $(100 \mathrm{~ms})$ to detect returning PUs (emulated by a signal generator emitting signals on one of the WiFi bands). The sensing-period adaptation, however, can also be implemented by reserving a quiet period (at MAC level) every $n$ frames and adapting $n$ accordingly.

\subsection{Application to Licensed Bands}

The proposed sensing-period optimization scheme is designed to be adaptive to time-varying channel environments in order to track diverse channel-usage behavior of heterogeneous applications (for example, voice, video streaming, Web browsing, etc.). For example, if there exists a long ON period on a TV band, the proposed adaptive estimation technique with a moving time-window will accumulate $1(\mathrm{ON})$ samples and its estimates of mean $\mathrm{ON} /$ OFF periods will eventually converge to the maximum/ minimum values. As a result, the channel's sensing period is adapted to a larger value since the channel is not likely to be available.

In case a licensed band has very short ON/OFF periods, spectrum agility may not bring much benefit due to high sensing overhead to track the fast ON/OFF state transitions.
Our goal in this paper, however, is to provide a general sensing framework that can be applied to any application on any band by providing an adaptive sensing method and estimation technique.

\section{Related Work}

There have been a limited number of publications on MAClayer sensing. Chou [12] proposed a proactive sensing algorithm with nonadaptive and randomly chosen sensing periods in which he did not consider how to maximize the discovery of opportunities. Zhao et al. [24] proposed a Decentralized Cognitive MAC (DC-MAC) with reactive sensing focusing on slotted-time CSMA-based channel access with synchronized slot information. Sankaranarayanan et al. [25] proposed an Ad-hoc Secondary system MAC (AS-MAC), which is a proactive scheme with slotted-time-based channel access. However, [24] and [25] did not consider the inherent trade-off between sensing overhead and discovery of opportunities. Although Liu and Shankar N. [26] pointed out the impact of number of samples on confidence of estimation, they did not recognize the importance of the upper bound approach in adapting sensing periods, as discussed in Section 6.

\section{Conclusion and Future Work}

We studied two optimization problems: sensing-period adaptation and optimal sensing-sequencing at channel switching. Both solutions are used to discover spectrum opportunities more efficiently by considering the underlying ON-OFF channel-usage patterns. The proposed scheme strives to discover as many utilizable spectrum opportunities as possible. A channel-usage pattern estimation technique was also proposed by deriving ML estimators and their confidence intervals. The simulation results demonstrated the advantages of the proposed algorithms such as robustness of parameter estimation, a larger amount of discovered channel availability, and a smaller channelswitching delay.

In the future, we would like to develop a more practical algorithm that can recognize higher layers' bandwidth requirements so that the sensing periods can be intelligently adapted to meet the requirements. This would be done with a cross-layer design approach, and it will make the proposed sensing scheme more effective in real CRNs.

\section{ACKNOWLEDGMENTS}

The work reported in this paper was supported in part by the US National Science Foundation under Grant CNS 0519498 and by Intel Corp.

\section{REFERENCES}

[1] Spectrum Policy Task Force Report, ET Docket No. 02-135, Nov. 2002.

[2] C. Bergstrom, S. Chuprun, and D. Torrieri, "Adaptive Spectrum Exploitation Using Emerging Software Defined Radios," Proc. IEEE Radio and Wireless Conf., pp. 113-116, Aug. 1999.

[3] S. Haykin, "Cognitive Radio: Brain-Empowered Wireless Comm.," IEEE J. Selected Areas in Comm., vol. 23, no. 2, pp. 201220, Feb. 2005. 
[4] W.D. Horne, "Adaptive Spectrum Access: Using the Full Spectrum Space," Proc. Int'l Symp. Advanced Radio Technologies, Mar. 2003.

[5] Vision RFC, http://www.ir.bbn.com/projects/xmac/rfc/rfcvision.pdf, 2007

[6] Arch. RFC, http://www.ir.bbn.com/projects/xmac/rfc/rfcaf.pdf, 2007.

[7] Facilitating Opportunities for Flexible, Efficient, and Reliable Spectrum Use Employing Cognitive Radio Technologies, FCC Notice of Proposed Rulemaking and Order-ET Docket 03-108, Dec. 2003.

[8] D. Cabric, S.M. Mishra, and R.W. Brodersen, "Implementation Issues in Spectrum Sensing for Cognitive Radios," Proc. Asilomar Conf. Signals, Systems, and Computers, pp. 772-776, Nov. 2004.

[9] H. Tang, "Some Physical Layer Issues of Wide-Band Cognitive Radio Systems," Proc. IEEE Int'l Symp. Dynamic Spectrum Access Networks (DySPAN '05), pp. 151-159, Nov. 2005.

[10] S. Shankar, C. Cordeiro, and K. Challapali, "Spectrum Agile Radios: Utilization and Sensing Architectures," Proc. IEEE Int'l Symp. Dynamic Spectrum Access Networks (DySPAN '05), pp. 160169, Nov. 2005.

[11] IEEE 802.22 Working Group on Wireless Regional Area Networks, http:/ / www.ieee802.org/22/, 2008.

[12] C.-T. Chou, "Adaptive Quality-of-Service Provisioning in Wireless/Mobile Networks," PhD dissertation, Univ. of Michigan, 2004.

[13] T. Keller and L. Hanzo, "Adaptive Multicarrier Modulation: A Convenient Framework for Time-Frequency Processing in Wireless Communications," Proc. IEEE, vol. 88, no. 5, pp. 611-640, May 2000.

[14] R. Rajbanshi, Q. Chen, A.M. Wyglinski, G.J. Minden, and J.B. Evans, "Quantitative Comparison of Agile Modulation Techniques for Cognitive Radio Transceivers," Proc. Fourth IEEE Consumer Comm. and Networking Conf. Workshop Cognitive Radio Networks, pp. 1144-1148, Jan. 2007.

[15] H. Kim, C. Cordeiro, K. Challapali, and K.G. Shin, "An Experimental Approach to Spectrum Sensing in Cognitive Radio Networks with Off-the-Shelf IEEE 802.11 Devices," Proc. Fourth IEEE Consumer Comm. and Networking Conf. Workshop Cognitive Radio Networks, pp. 1154-1158, Jan. 2007.

[16] C. Cordeiro, K. Challapali, and M. Ghosh, "Cognitive PHY and MAC Layers for Dynamic Spectrum Access and Sharing of TV Bands," Proc. First Int'l Workshop Technology and Policy for Accessing Spectrum (TAPAS '06), Aug. 2006.

[17] A. Ghasemi and E.S. Sousa, "Collaborative Spectrum Sensing for Opportunistic Access in Fading Environments," Proc. IEEE Int'l Symp. Dynamic Spectrum Access Networks (DySPAN '05), pp. 131136, Nov. 2005

[18] G. Ganesan and Y. Li, "Cooperative Spectrum Sensing in Cognitive Radio Networks," Proc. IEEE Int'l Symp. Dynamic Spectrum Access Networks (DySPAN '05), pp. 137-143, Nov. 2005.

[19] E. Visotsky, S. Kuffner, and R. Peterson, "On Collaborative Detection of TV Transmissions in Support of Dynamic Spectrum Sharing," Proc. IEEE Int'l Symp. Dynamic Spectrum Access Networks (DySPAN '05), pp. 338-345, Nov. 2005.

[20] D.R. Cox, Renewal Theory. Butler and Tanner, 1967.

[21] S.M. Ross, Stochastic Processes. John Wiley \& Sons, 1983.

[22] C. Cordeiro, K. Challapali, D. Birru, and S. Shankar, "IEEE 802.22: The First Worldwide Wireless Standard Based on Cognitive Radios," Proc. IEEE Int'l Symp. Dynamic Spectrum Access Networks (DySPAN '05), pp. 328-337, Nov. 2005.

[23] C.R. Stevenson, C. Cordeiro, E. Sofer, and G. Chouinard, "Functional Requirements for the 802.22 WRAN Standard," IEEE 802.22 Draft, Sept. 2005.

[24] Q. Zhao, L. Tong, and A. Swami, "Decentralized Cognitive MAC for Dynamic Spectrum Access," Proc. IEEE Int'l Symp. Dynamic Spectrum Access Networks (DySPAN '05), pp. 224-232, Nov. 2005.

[25] S. Sankaranarayanan, P. Papadimitratos, and A. Mishra, "A Bandwidth Sharing Approach to Improve Licensed Spectrum Utilization," Proc. IEEE Int'l Symp. Dynamic Spectrum Access Networks (DySPAN '05), pp. 279-288, Nov. 2005.

[26] X. Liu and S. Shankar N., "Sensing-Based Opportunistic Channel Access," J. Mobile Networks and Applications, vol. 11, no. 4, pp. 577591, Aug. 2006

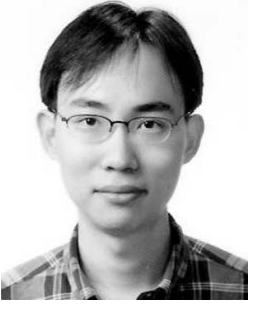

Hyoil Kim received the BS degree in electrical engineering from Seoul National University, Korea, in 1999 and the MS degree from the University of Michigan in 2005 . He is currently a PhD candidate in the Department of Electrical Engineering and Computer Science (EECS), University of Michigan, Ann Arbor. Since 2004, he has been a research assistant in the RealTime Computing Laboratory, EECS Department. His research interests include Dynamic Spectrum Access (DSA) and cognitive radio networks, spectrum sensing and opportunity discovery, and wireless spectrum resource allocation/ management. He received the Korea Science and Engineering Foundation (KOSEF) Scholarship from 2003 to 2005 and, currently, the Samsung Scholarship Foundation (formerly known as the Samsung Lee Kun Hee Scholarship Foundation) in 2005.

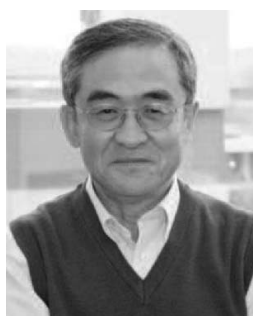

Kang G. Shin received the BS degree in electronics engineering from Seoul National University, Korea, in 1970 and the MS and $\mathrm{PhD}$ degrees in electrical engineering from Cornell University, Ithaca, New York, in 1976 and 1978, respectively. From 1978 to 1982, he was on the faculty of the Rensselaer Polytechnic Institute, Troy, New York. He has held visiting positions at the US Airforce Flight Dynamics Laboratory, AT\&T Bell Laboratories, the Computer Science Division, Department of Electrical Engineering and Computer Science (EECS), University of California, Berkeley, the International Computer Science Institute, Berkeley, California, the IBM T.J. Watson Research Center, the Software Engineering Institute, Carnegie Mellon University, and HP Research Laboratories. He was the chair of the Computer Science and Engineering Division, EECS Department, University of Michigan, for three years beginning January 1991. He is the Kevin and Nancy O'Connor professor of Computer Science and founding director of the Real-Time Computing Laboratory, Department of Electrical Engineering and Computer Science, University of Michigan, Ann Arbor. His current research focuses on QoS-sensitive networking and computing, as well as embedded real-time OS, middleware, and applications, all with an emphasis on timeliness and dependability. He has supervised the completion of $57 \mathrm{PhD}$ dissertations and was the author or coauthor of more than 650 technical papers (235 of which are in archival journals) and numerous book chapters on distributed real-time computing and control, computer networking, faulttolerant computing, and intelligent manufacturing. He was a coauthor (jointly with C.M. Krishna) of the textbook Real-Time Systems (McGraw Hill, 1997). He was also a coauthor of papers with his students which received Best Student Paper Awards from the 1996 IEEE Real-Time Technology and Application Symposium and the 2000 UNSENIX Technical Conference. He is a fellow of the IEEE and the ACM and a member of the Korean Academy of Engineering. He has received a number of best paper awards, including the IEEE Communications Society William R. Bennett Prize Paper Award in 2003, the Best Paper Award from IWQoS in 2003, and an Outstanding IEEE Transactions on Automatic Control Paper Award in 1987. He has also received several institutional awards, including the Research Excellence Award in 1989 the Outstanding Achievement Award in 1999, the Service Excellence Award in 2000, the Distinguished Faculty Achievement Award in 2001 , and the Stephen Attwood Award in 2004 from the University of Michigan, a Distinguished Alumni Award of the College of Engineering, Seoul National University in 2002, a 2003 IEEE RTC Technical Achievement Award, and a 2006 Ho-Am Prize in Engineering. He has served as the general chair for the Third ACM/Usenix International Conference on Mobile Systems, Applications, and Services (MobiSys '05), the 2000 IEEE Real-Time Technology and Applications Symposium, and the 1987 RTSS, as a program cochair for the 1992 International Conference on Parallel Processing, and as the program chair of the 1986 IEEE RealTime Systems Symposium (RTSS) in addition to serving on numerous technical program committees. He chaired the IEEE Technical Committee on Real-Time Systems from 1991 to 1993 and was a distinguished visitor of the IEEE Computer Society. He was the guest editor of the 1987 August special issue of the IEEE Transactions on Computers on Real-Time Systems, an editor of IEEE Transactions on Parallel and Distributed Computing, and an area editor of the International Journal of Time-Critical Computing Systems, Computer Networks, and ACM Transactions on Embedded Systems. 